\title{
Generation of attosecond electron bunches in a laser-plasma accelerator using a plasma density upramp
}

\author{
M.K. Weikum ${ }^{\mathrm{a}, \mathrm{b}, *}$, F.Y. Li ${ }^{\mathrm{b}}$, R.W. Assmann ${ }^{\mathrm{a}}$, Z.M. Sheng ${ }^{\mathrm{b}, \mathrm{c}}$, D. Jaroszynski ${ }^{\mathrm{b}}$ \\ ${ }^{a}$ Deutsches Elektronensynchrotron (DESY), 22607 Hamburg, Germany \\ ${ }^{b}$ Department of Physics, University of Strathclyde, G4 ONG Glasgow, United Kingdom \\ ${ }^{c}$ Laboratory for Laser Plasmas and Department of Physics and Astronomy, Shanghai Jiao Tong University, Shanghai 200240, China
}

\begin{abstract}
Attosecond electron bunches and attosecond radiation pulses enable the study of ultrafast dynamics of matter in an unprecedented regime. In this paper, the suitability for the experimental realization of a novel scheme producing subfemtosecond duration electron bunches from laser-wakefield acceleration in plasma with self-injection in a plasma upramp profile has been investigated. While it has previously been predicted that this requires laser power above a few hundred terawatts typically, here we show that the scheme can be extended with reduced driving laser powers down to tens of terawatts, generating accelerated electron pulses with minimum length of around 166 attoseconds and picocoulombs charge. Using particle-in-cell simulations and theoretical models, the evolution of the accelerated electron bunch within the plasma as well as simple scalings of the bunch properties with initial laser and plasma parameters are presented.
\end{abstract}

Keywords: Ultrashort electron bunch; Laser wakefield; Wave breaking; Inhomogeneous plasma; Upramp

\section{Introduction}

Current techniques for the observation of ultrafast pro- $^{29}$ cesses are limited in temporal resolution due to Auger de- ${ }^{30}$ cay and similar mechanisms changing the electronic structure of an atom within time scales of up to few femtoseconds. The development of electron and light pulses in the attosecond regime is therefore necessary in order to overcome this boundary and make the real-time study of 34 these phenomena through both the structural and electronic evolution of their constituents possible at full reso- ${ }^{35}$ lution 1, 2.

While conventional accelerators are generally restricted ${ }^{37}$ to pulse lengths of tens to hundreds of femtoseconds, laser- ${ }^{38}$ plasma accelerators have recently been able to demon- ${ }^{39}$ strate the production of electron bunches down to few ${ }^{40}$ fs RMS (root-mean-square) bunch duration 3, 4, Ex- ${ }^{41}$ periments with inverse Free-Electron Lasers further re- ${ }^{42}$ ported the generation of electron pulses of few hundred ${ }^{43}$ attoseconds length, however, only as part of longer bunch ${ }^{44}$ trains which has significantly limited their applicability ${ }^{45}$ so far [5]. For the acceleration of isolated, sub-fs elec- ${ }^{46}$ tron beams, a number of schemes has been proposed in recent years, all majorly based on one of three principal ${ }^{47}$ setups: laser plasma acceleration with ultrathin overdense targets [6, 7, 8] or droplets [9, 10], direct laser accelera- ${ }_{48}$ tion in vacuum [11, 12] and pulse compression of conven- ${ }_{49}$ tionally accelerated electron beams [13]. Although for the ${ }_{50}$

* Corresponding Author at: DESY, Bdg. 30b, Notkestr. 85, 22607 Hamburg, Germany. Tel.: +49 4089983851.

Email address: maria.weikum@desy.de (M.K. Weikum) latter experimental facilities are scheduled to be set up in the next years, so far all of these techniques are based on simulations and theoretical work most facing a number of technical challenges, such as very high laser contrast ratios, low timing jitters or the need for high power $\mathrm{THz}$ sources, yet to be overcome.

\section{Injection with a plasma density upramp}

In this paper, we have thus investigated a different approach for producing attosecond electron bunches, through laser-plasma acceleration with an upramp density transition, originally suggested and described in detail by Li et al [14. As shown in Fig. 1] a broad, high intensity laser pulse is propagated through a plasma with density profile consisting of an upramp from vacuum of length $R$ followed by a plateau of constant density $n_{0}$.

The effect of the ramped shape on the generated nonlinear plasma wave is two-fold: on the one hand, the variation in density affects the plasma wavelength and hence the wave phase velocity $\beta_{p h}=v_{p h} / c$ quantified as

$$
\beta_{p h}=\frac{\beta_{g r}}{1-\frac{|\xi|}{2 n} \frac{d n}{d x}}
$$

where $\beta_{g r}=v_{g r} / c$ is the normalised laser group velocity, $n$ the local background plasma density and $\xi=\mathrm{x}-\beta_{\text {gr }} c t$ the distance from the local position $x$ to the laser pulse [14. A positive density gradient thus leads to an increase of the phase velocity $\beta_{p h}$ above 1 (see dashed line, Fig. 1) and consequently a suppression of wave breaking and electron 


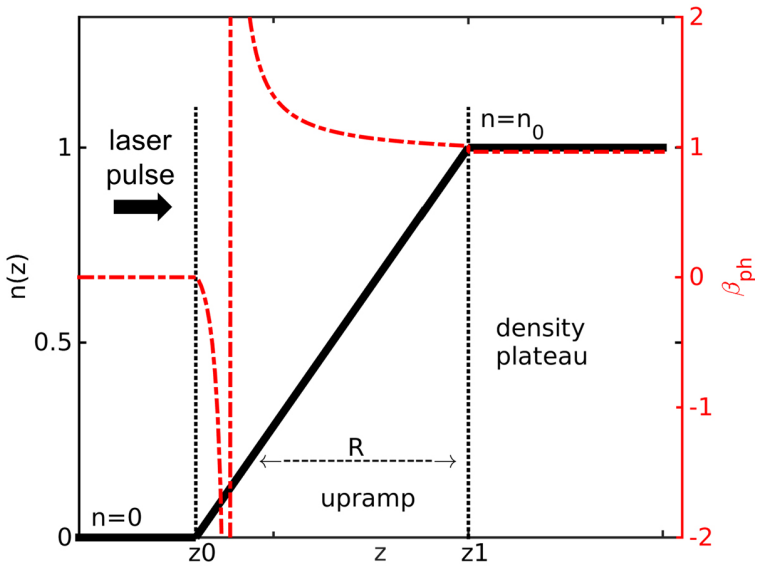
the density-dependent plasma wave phase velocity (dashed line) as a function of position in the laser propagation direction $z$. The phase velocity is derived from Eq. (1) for a distance $|\xi|$ of one nonlin- ${ }^{101}$ ear plasma wavelength $\lambda_{n p}=\lambda_{p}\left(1+a_{0}^{2} / 2\right)^{1 / 4}$ behind the laser102 pulse with $\lambda_{p}=(2 \pi c / e) \sqrt{\epsilon_{0} m_{e} / n}$ defined as the linear plasma wave-103 length, and $e, m_{e}$ as the electron charge and mass, respectively. ${ }_{104}$

trapping, as long as the density gradient is large enough. ${ }^{106}$ Note that the phase velocity becomes negative represent- ${ }^{107}$ ing backward moving plasma waves for very small densities ${ }^{108}$ with $|\xi| /(2 n) d n / d x>1$ before approaching a singularity ${ }^{109}$ with $k_{p} \rightarrow 0$, as seen at the beginning of the ramp in Fig. ${ }_{111}^{110}$ 1.

The second effect of the density transition is on the ${ }^{112}$ width of the plasma wave crests which Bulanov et al [15 ${ }_{114}^{113}$ derived as

$$
\delta n=\left[\frac{2\left(\beta_{p h}-\beta_{m}\right)^{3}}{\beta_{m}\left(1-\beta_{m}^{2}\right)^{3 / 2}}\right]^{1 / 2}
$$

where $\beta_{m}$ is the maximum electron quiver velocity in the ${ }_{110}^{11}$ plasma wave. With the phase velocity decreasing towards ${ }^{120}$ the limit of $\beta_{g r}$ on the density ramp, the density peaks ${ }^{120}$ of the wave crests hence narrow down quickly to values of ${ }_{122}^{122}$ tens to hundreds of attoseconds width on the ramp.

At the transition of the inhomogeneous region to the plasma plateau the density gradient drops to zero, so that ${ }_{125}$ the condition for wave breaking suppression given in Eq. ${ }_{126}$ (1) is no longer effective. If the laser intensity is cho- ${ }^{127}$ sen such that the plasma wave is highly nonlinear, a large fraction of electrons from the first wave crest is injected at this point into the wakefield to be accelerated further in the homogeneous plasma. Due to the sudden nature of ${ }_{131}^{130}$ the trapping process, the shape of the injected electrons from the wave crest is very well preserved hence forming ${ }_{132}^{132}$ a broad attosecond duration electron sheet.

While Li et al studied this phenomenon at high laser ${ }^{134}$ intensities of ca. $5 \times 10^{19} \mathrm{Wcm}^{-2}$ [14], corresponding to ${ }_{136}^{135}$ $a_{0}=\left(e E_{L}\right) /\left(m_{e} c \omega\right)=6$ (with $\omega$ the laser frequency), we ${ }^{136}$ have focused our research on extending this initial regime to lower values of $a_{0}$, both showing the scheme's tunabil-

by

stre strength for injection is lowered from around $a_{0}=6$, as originally predicted for $n=0.03-0.04 n_{c r}$ (with $n_{c r}$ the critical plasma density for the laser wavelength $\lambda$ ) [14, to $a_{0}=2.5$ -

ity and providing experimentally more easily achievable parameters. With particle-in-cell simulations it was thus possible to demonstrate a decrease of the required laser intensity by a factor of 4 through optimization of the laser and plasma properties.

\section{One-Dimensional Simulations}

Figures 2 and 3 depict the results of one-dimensional particle-in-cell (PIC) simulations performed with the code OSIRIS 16 to study the dependence of the electron bunch properties from injection in the previously described upramp setup on the laser strength, pulse length and plasma plateau background density. The drive laser pulse was assumed to have a wavelength of $1 \mu \mathrm{m}$ with a Gaussian transverse and a $\sin ^{2}$ longitudinal profile. A simulation box of size 145 to $200 \mu \mathrm{m}$ was used with a resolution $5 \mathrm{~nm}$ and 30 particles per cell. As shown in Fig. 2 . employing a very short laser pulse the threshold laser 3 corresponding to an intensity of around $8.6 \times 10^{18} \mathrm{Wcm}^{-2}$ to $1.2 \times 10^{19} \mathrm{Wcm}^{-2}$, respectively. Longer electron bunch durations and lower bunch charge are found under these conditions, as the strength of the wave breaking process is decreased. For $a_{0}<2.5$ as well as for $a_{0}<3$ at a density of $3 \% n_{c r}$ the accelerating field is below the wave breaking threshold and hence no trapping takes place at all.

Further tuning of the electron beam properties can be achieved through variation of, for example, the laser pulse length, as seen in Fig. 3(a). Here, a minimal bunch duration is found for a pulse length roughly matched to the nonlinear plasma wavelength; the charge of the beam, being proportional to the duration, behaves very similarly and exhibits a low charge region around laser pulse length values with minimal electron bunch duration. Figure 3(b) shows that the electron bunch length decreases with plateau background density due to the $\mathrm{n}$-dependence of both the plasma phase velocity (see Eq. (10) and the maximum electron velocity. For larger plasma densities, however, such as here $n=0.07 n_{c r}$, dephasing occurs very shortly after injection spoiling the beam quality through stretching of the pulse. The bunch charge increases at higher density, as the number of electrons in the plasma wave crests available for trapping rises.

It should be noted that, although the presented 1D results provide a good indication of the useful input parameter regions, they do not provide a reliable quantitative description of the injection scheme, as transverse effects, such as laser self-focusing and transverse electron injection by the ponderomotive force are excluded in one-dimensional PIC simulations. The absolute charge and hence beamloading effects are also not calculated fully in the 1D PIC code and are therefore underestimated. 

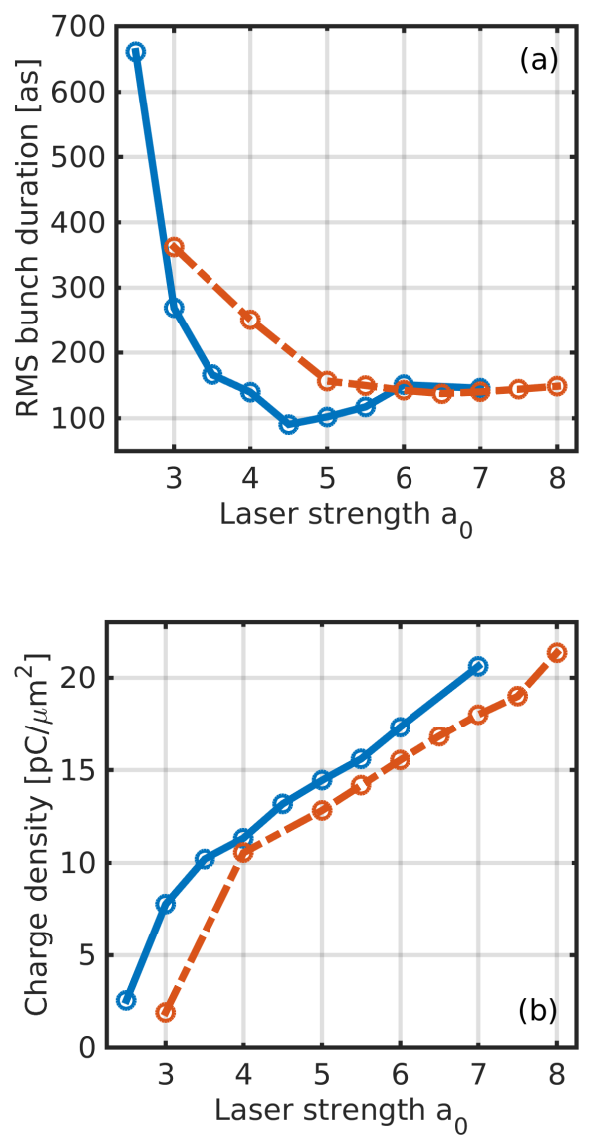

Figure 2: RMS bunch duration (a) and charge density (b) of the injected electron bunch as a function of laser strength $a_{0}$ from 1D PIC simulations. The laser pulse length was taken to be $3 \mu \mathrm{m}$ (FWHM), the length of the upramp $R=45 \mu \mathrm{m}$ and the background plateau density $n_{0}=3.36 \times 10^{25} \mathrm{~m}^{-3}$ (dashed line) and $4.48 \times 10^{25} \mathrm{~m}^{-3}$ (solid line) respectively.

\section{Three-Dimensional Simulations}

For a more comprehensive understanding of the injec- ${ }^{159}$ tion scheme, including the development of the accelerated ${ }^{160}$ electron beam throughout longer propagation in the ho- ${ }^{161}$ mogeneous plasma plateau, 3D PIC simulations have been ${ }^{162}$ performed with OSIRIS using a comoving window of $\mathrm{di}^{163}$ mensions 23x110x $110 \mu \mathrm{m}^{3}$ and cell size $16.4 \times 157 \times 157 \mathrm{~nm}^{3} .{ }^{164}$ A number of 8 particles per cell is employed and the laser ${ }^{165}$ is assumed to possess the same properties as in the $1 \mathrm{D}^{166}$ studies with a laser spot size of $30 \mu \mathrm{m}$ (FWHM), a peak ${ }^{167}$ intensity of $1.2 \times 10^{19} \mathrm{Wcm}^{-2}$ and pulse duration of $3 \mu m^{168}$ (FWHM) in this case.

Figure 4 details how the density structure of the plasma ${ }^{170}$ develops behind the laser pulse. In each case, the laser ${ }^{171}$ pulse position is marked by a solid line, while the accel- ${ }^{172}$ erated electron bunch is identified by a dashed line rep- ${ }^{173}$ resenting the bunch density at $\mathrm{x}=\mathrm{y}=0$. Noting the differ ${ }^{-174}$ ent spatial scales for the longitudinal and transverse direc- ${ }^{175}$ tions, Fig. 4(a) shows the first plasma wave crest behind ${ }^{176}$ the laser pulse appearing as an ultrathin electron sheet. ${ }^{177}$ In Fig. 4(b) a large fraction of these electrons has been ${ }^{178}$
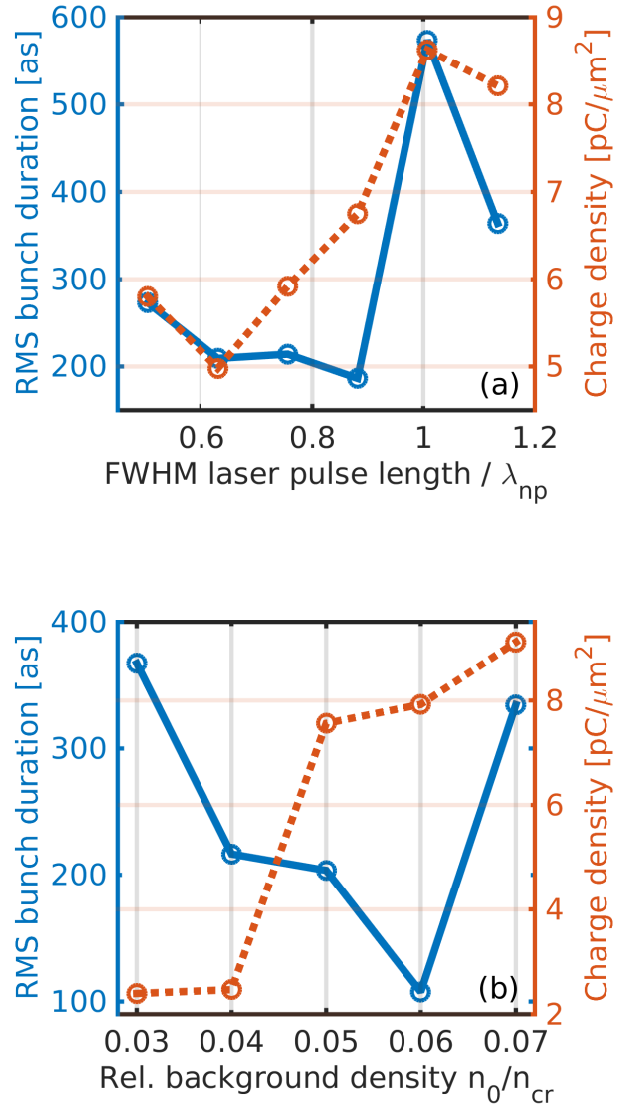

Figure 3: RMS bunch duration (solid line) and charge density (dotted line) of the injected electron bunch as a function of the laser pulse length (a) and the plateau plasma density $n_{0}$ (b) from $1 \mathrm{D}$ PIC simulations. In (a) the background density was taken to be $n_{0}=5.6 \times 10^{25} \mathrm{~m}^{-3}$ with a ramp length of $R=100 \mu \mathrm{m}$. In (b) the laser pulse length is $4 \mu \mathrm{m}$ (FWHM) with an upramp of length $R=45 \mu \mathrm{m}$. In both cases $a_{0}=3$.

injected into the remaining wakefield with this preserved ultrathin form. A strong evolution of the electron beam shape is observed after in Fig. 4(c) and (d) due to strong transverse wakefields as well as beamloading because of the very high initial peak current of 1.6 MA. Within a distance of ca. $120 \mu \mathrm{m}$, the electron bunch has first focused longitudinally and transversely (Fig. 4(c)) before defocusing and losing more than $85 \%$ of its initial charge (Fig. 4 (d)). At this point, the laser pulse has been depleted strongly, while the electron beam has reached a mean energy of around $45 \mathrm{MeV}$. Note that the bunch itself is defined in this case as those electrons having energy above $5.12 \mathrm{MeV}$, positive forward momentum and forming a region of charge equal or larger than $25 \%$ of the maximum charge. The last criterion was chosen particularly to distinguish the beam from the background plasma where small groups of electrons can be accelerated to low energies continuously due to the nonlinearity of the wakefield.

Although the effective electron bunch duration is increased by the curvature of the beam, a minimum RMS 


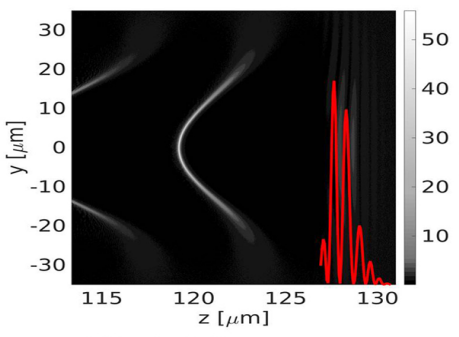

(a) $\mathrm{T}=378 \mathrm{fs}$

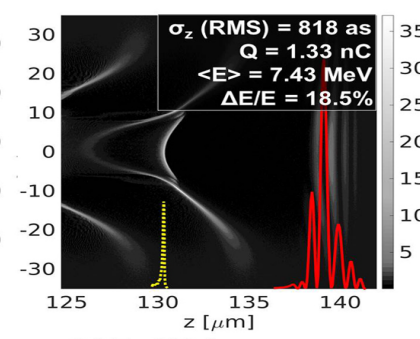

(b) $\mathrm{T}=416 \mathrm{fs}$

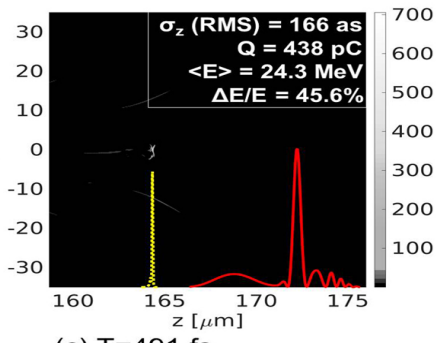

(c) $\mathrm{T}=491 \mathrm{fs}$

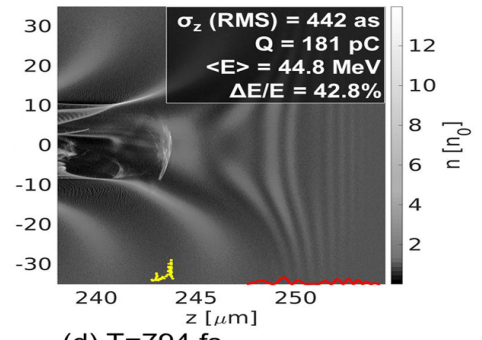

(d) $\mathrm{T}=794 \mathrm{fs}$

Figure 4: Temporal development of the plasma density structure around the accelerated electron bunch before (a) and after (b)-(d) injection. The following simulation parameters are assumed: a driving laser with strength $a_{0}=3$, pulse length $3 \mu \mathrm{m}$ (FWHM) and spot size $30 \mu \mathrm{m}$ (FWHM), plasma with plateau density $n / n_{c r}=0.03=3.36 \times 10^{25} \mathrm{~m}^{-3}$ and an upramp of length $R=100 \mu \mathrm{m}$ (ramp end at $\left.\mathrm{z}=123 \mu \mathrm{m}\right)$. The solid line further describes the laser electric field, whereas the dotted line is the density of the injected bunch (divided by 15 in (c)) at $\mathrm{x}=\mathrm{y}=0$.

length of 166 attoseconds for the whole bunch, and 127 attoseconds within the central $92 \times 92 \mathrm{~nm}^{2}$ transverse region of the beam, is found for a point of strong beam focus around $40 \mu \mathrm{m}$ after the end of the upramp. Both the ultrashort bunch length and the relatively large charge of few hundred pC hence provide very useful conditions for a potential use of these electron bunches for radiation generation. One aspect to improve, on the other hand, is the large beam energy spread of few tens of percent; this is caused by a combination of continuous injection of electrons just after the ramp end and strong beamloading fields decelerating the back of the beam.

Figure 5 compares the outcome presented here with results from published simulations as well as experimental bunch length measurements in different setups. As seen on the semilogarithmic plot, both simple self-injection 4, 17, 18 and more complex controlled injection schemes [3, such as colliding pulse injection, produce electron beams with duration of a few femtoseconds. Much shorter pulses are generated as reported by Li et al [14, however, with laser intensities 8 to 50 times larger than in the experimental cases. The regime we have described in this paper thus represents not only a region so far unexplored experimentally, but also one providing a good compromise between ultrashort electron bunch duration and required ${ }_{218}$ laser intensity.

\section{Summary and Outlook}

Particle-in-cell simulations have shown that sub-fs elec- ${ }^{22}$ tron pulses can be produced and accelerated in a laser 222 wakefield driven along an upramp density profile with $\mathrm{a}_{223}$ minimum laser intensity lower than originally predicted $\mathrm{in}_{224}$ 14] by a factor of around 4. 1D scalings demonstrate $\mathrm{a}_{225}$ dependence of the injected beam properties on not only ${ }_{226}$ the laser strength $a_{0}$, but also the laser pulse length $\operatorname{and}_{227}$ background density. Minimum bunch lengths of below $200_{228}$ attoseconds are thus estimated despite a relatively strong 229 evolution of the bunch within the plasma. Future efforts ${ }_{230}$ will focus on stabilising the electron beam and reducing ${ }_{231}$ its energy spread; in this context it is planned to investi- ${ }_{232}$

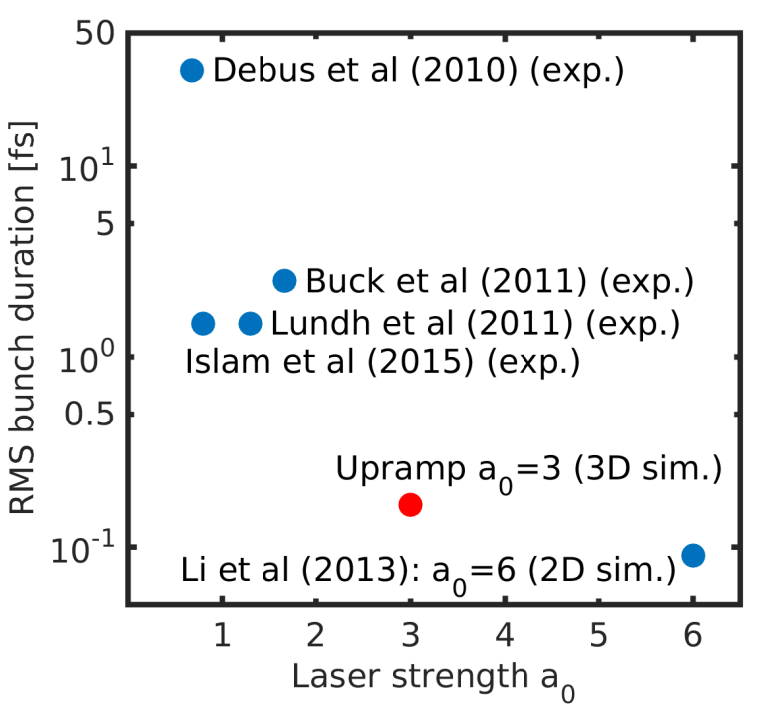

Figure 5: Comparison of the minimum RMS electron bunch length as a function of laser strength $a_{0}$ from experiments (exp.) and simulation (sim.) data in different setups. For the upramp simulation for $a_{0}=3$ the same parameters as in Fig. 4 are used. Note that the graph is in semilogarithmic scale.

gate more complex density profiles in order to regulate the trapping time and propagate the beam more stably after the end of the plasma ramp.

\section{Acknowledgements}

We would like to acknowledge the OSIRIS Consortium, consisting of UCLA and IST (Lisbon, Portugal) for the use of OSIRIS and providing access to the OSIRIS framework. Further acknowledgements go to the Juelich Supercomputing Centre and their grant for computing time on JUQUEEN under Project No. HHH23 as well as the ARCHER UK National Supercomputing Centre for computing time under project E281. Many thanks also to Julia Grebenyuk and the FLA Plasma Group at DESY for their support and helpful discussions. This work is supported by a Leverhulme Trust Research Grant. 


\section{References}

[1] L. Young, E. P. Kanter, B. Krässig, Y. Li, A. M. March, S. T. Pratt, R. Santra, S. H. Southworth, N. Rohringer, L. F.306 Dimauro, G. Doumy, C. A. Roedig, N. Berrah, L. Fang, ,307 M. Hoener, P. H. Bucksbaum, J. P. Cryan, S. Ghimire, J. M.308 Glownia, D. A. Reis, J. D. Bozek, C. Bostedt, M. Messer-309 schmidt, Femtosecond electronic response of atoms to ultra-310 intense X-rays., Nature 466 (2010) 56-61. doi:10.1038/311 nature09177

[2] P. Corkum, F. Krausz, Attosecond science, Nat. Phys. 3 (2007) $)_{313}$ 381-387. doi:10.1038/nphys620

[3] O. Lundh, J. Lim, C. Rechatin, L. Ammoura, A. Ben-Ismaïl,315 X. Davoine, G. Gallot, J.-P. Goddet, E. Lefebvre, V. Malka,316 J. Faure, Few femtosecond, few kiloampere electron bunch pro-317 duced by a laserplasma accelerator, Nat. Phys. 7 (2011) 219-318 222. doi: $10.1038 /$ nphys 1872

[4] A. Buck, M. Nicolai, K. Schmid, C. M. S. Sears, A. Sävert, J. M. ${ }_{320}$ Mikhailova, F. Krausz, M. C. Kaluza, L. Veisz, Real-time ob-321 servation of laser-driven electron acceleration, Nat. Phys. 7 (7) 322 (2011) 543-548. doi:10.1038/nphys1942

[5] C. M. S. Sears, E. Colby, R. Ischebeck, C. McGuinness, J. Nel-324 son, R. Noble, R. H. Siemann, J. Spencer, D. Walz, T. Plettner,325 R. L. Byer, Production and characterization of attosecond electron bunch trains, Phys. Rev. Spec. Top. - Accel. Beams 11 (6) (2008) 061301. doi:10.1103/PhysRevSTAB.11.061301

[6] H. C. Wu, J. Meyer-Ter-Vehn, J. Fernández, B. M. Hegelich, Uniform laser-driven relativistic electron layer for coherent Thomson scattering, Phys. Rev. Lett. 104 (23) (2010) 234801. doi:10.1103/PhysRevLett.104.234801

[7] V. V. Kulagin, V. A. Cherepenin, M. S. Hur, H. Suk, Theoretical investigation of controlled generation of a dense attosecond relativistic electron bunch from the interaction of an ultrashort laser pulse with a nanofilm, Phys. Rev. Lett. 99 (12) (2007) 124801. doi:10.1103/PhysRevLett.99.124801.

[8] Y. Y. Ma, Z. M. Sheng, Y. T. Li, W. W. Chang, X. H. Yuan, M. Chen, H. C. Wu, J. Zheng, J. Zhang, Dense quasimonoenergetic attosecond electron bunches from laser interaction with wire and slice targets, Phys. Plasmas 13 (11) (2006) 110702. doi:10.1063/1.2388958

[9] L. Di Lucchio, P. Gibbon, Relativistic attosecond electron bunch emission from few-cycle laser irradiated nanoscale droplets, Phys. Rev. Spec. Top. - Accel. Beams 18 (2) (2015) 023402. doi:10.1103/PhysRevSTAB.18.023402

[10] T. V. Liseykina, S. Pirner, D. Bauer, Relativistic attosecond electron bunches from laser-illuminated droplets, Phys. Rev. Lett. 104 (9) (2010) 095002. doi:10.1103/PhysRevLett.104. 095002

[11] A. Sell, F. X. Kärtner, Attosecond electron bunches accelerated and compressed by radially polarized laser pulses and soft-x-ray pulses from optical undulators, J. Phys. B At. Mol. Opt. Phys. 47 (2014) 015601. doi:10.1088/0953-4075/47/1/015601

[12] G. V. Stupakov, M. S. Zolotorev, Ponderomotive laser acceleration and focusing in vacuum for generation of attosecond electron bunches, Phys. Rev. Lett. 86 (23) (2001) 5274-5277. doi:10.1103/PhysRevLett.86.5274

[13] R. Assmann, C. Behrens, R. Brinkmann, U. Dorda, K. Flöttmann, B. Foster, J. Grebenyuk, M. Gross, I. Hartl, M. Hüning, F. Kärtner, B. Marchetti, Y. Nie, J. Osterhoff, A. Rühl, H. Schlarb, B. Schmidt, F. Stephan, A. S. Müller, M. Schuh, F. Grüner, B. Hidding, A. R. Maier, B. Zeitler, Sinbad - a proposal for a dedicated accelerator research facility at Desy, Proceedings of the 2014 International Particle Accelerator Conference TUPME047 (2014) 1466-1469.

[14] F. Y. Li, Z. M. Sheng, Y. Liu, J. Meyer-Ter-Vehn, W. B. Mori, W. Lu, J. Zhang, Dense attosecond electron sheets from laser wakefields using an up-ramp density transition, Phys. Rev. Lett. 110 (13) (2013) 135002. doi:10.1103/PhysRevLett.110. 135002

[15] S. Bulanov, N. Naumova, F. Pegoraro, J. Sakai, Particle injection into the wave acceleration phase due to nonlinear wake wave breaking, Phys. Rev. E 58 (5) (1998) R5257-R5260. doi:10.1103/PhysRevE.58.R5257

[16] R. Fonseca, L. Silva, F. Tsung, V. Decyk, W. Lu, C. Ren, W. Mori, S. Deng, S. Lee, T. Katsouleas, J. Adam, OSIRIS: A three-dimensional, fully relativistic particle in cell code for modeling plasma based accelerators, Lecture Notes in Computer Science (2002) 342-351doi:10.1007/3-540-47789-6_36

17] M. R. Islam, E. Brunetti, R. P. Shanks, B. Ersfeld, R. C. Issac, S. Cipiccia, M. P. Anania, G. H. Welsh, S. M. Wiggins, A. Noble, R. A. Cairns, G. Raj, D. A. Jaroszynski, Near-threshold electron injection in the laser-plasma wakefield accelerator leading to femtosecond bunches, New J. Phys. 17 (9) (2015) 093033. doi: 10.1088/1367-2630/17/9/093033

[18] A. D. Debus, M. Bussmann, U. Schramm, R. Sauerbrey, C. D. Murphy, Z. Major, R. Hörlein, L. Veisz, K. Schmid, J. Schreiber, K. Witte, S. P. Jamison, J. G. Gallacher, D. A. Jaroszynski, M. C. Kaluza, B. Hidding, S. Kiselev, R. Heathcote, P. S. Foster, D. Neely, E. J. Divall, C. J. Hooker, J. M. Smith, K. Ertel, A. J. Langley, P. Norreys, J. L. Collier, S. Karsch, Electron bunch length measurements from laser-accelerated electrons using single-shot $\mathrm{THz}$ time-domain interferometry, Phys. Rev. Lett. 104 (8) (2010) 084802. doi:10.1103/PhysRevLett. 104.084802 